\title{
Alternating starvation of dislocations during plastic yielding in metallic nanowires
}

\author{
Ajing Cao, ${ }^{\mathrm{a}, *}$ Yueguang $\mathrm{Wei}^{\mathrm{a}}$ and Scott X. Mao ${ }^{\mathrm{b}}$ \\ ${ }^{a}$ State Key Laboratory of Nonlinear Mechanics, Institute of Mechanics, Chinese Academy of Sciences, \\ Beijing 100080, People's Republic of China \\ ${ }^{\mathrm{b}}$ Department of Mechanical Engineering and Materials Science, University of Pittsburgh, Pittsburgh, PA 15261, USA
}

Received 14 November 2007; revised 3 March 2008; accepted 7 March 2008

Available online 15 March 2008

\begin{abstract}
Using molecular dynamics simulations, we show that the mechanical deformation behaviors of single-crystalline nickel nanowires are quite different from their bulk counterparts. Correlation between the obtained stress-strain curves and the visualized defect evolution during deformation processes clearly demonstrates that a sequence of complex dislocation slip events results in a state of dislocation starvation, involving the nucleation and propagation of dislocations until they finally escape from the wires, so that the wires deform elastically until new dislocations are generated.

(C) 2008 Acta Materialia Inc. Published by Elsevier Ltd. All rights reserved.
\end{abstract}

Keywords: Metallic nanowires; Molecular dynamics; Dislocation starvation; Yielding

Due to the unique mechanical, thermal, electrical and optical properties, materials with nanometer-sized structure have attracted a great deal of interest as potential building blocks in nanoelectronic and nanoelectromechanical devices [1]. Many researchers have demonstrated, through both experiments and analysis, that the structure and properties of nanowires can be quite different from those of bulk materials due to the effect of the large surface to volume ratio [2-14].

Recently, Uchic et al. $[15,16]$ and Greer et al. $[17,18]$ reported that the plastic deformation behavior of single-crystalline sub-micropillars is dependent on the size of the pillar, even without a deformation gradient. More recently, a "mechanical annealing" test was used to demonstrate that dislocations can be swept out of the samples through the progressive activation and exhaustion of dislocation sources [19]. These promising results raise an important question: is the size effect still operative when the size of the pillar is reduced to under 10 nanometers?

Despite many efforts [6-14], a quantitative understanding of dislocation flow in nanoscale metals has remained elusive. In particular, the correlation of the dislocation flow with the stress-strain curve is of great interest, for the underlying mechanisms are typically

\footnotetext{
* Corresponding author. Present address: Department of Materials Science and Engineering, Johns Hopkins University. Tel.: +1 410243 4078; e-mail: ajcao@jhu.edu
}

represented by a mechanical response in macroscopic level experiments. An understanding of the post-yielding deformation mechanism is also of paramount importance. However, previous simulations have concentrated on the initial yielding behavior of metallic nanowires [911], while studies on the post-yielding are still lacking.

It is well-known that most conventional bulk metals only have their initial yield point represented by the stress-strain curve, their plastic flow being characterized as continuous dislocation movement after yielding. Here we show, by using molecular dynamics simulations, that metallic nanowires behave in an alternating dislocation starvation manner upon uniaxial compressive loading, which involves dislocation nucleation from free surfaces, dislocation travel across to the opposite side and then escape out of the wire system, so that continued elastic deformation is required to nucleate new dislocations.

We focus mainly on Ni[1 111$]$ nanowires with a nearly square cross-section. Nanowires with $(\overline{1} \overline{1} 2)$ and (1 $\overline{1} 0)$ side surfaces were created with their initial atomic position corresponding to the bulk face-centered-cubic (fcc) lattice. Three simulated nanowires were chosen, with transectional sizes of 4,8 and $16 \mathrm{~nm}$. The aspect ratio was fixed at 3:1. An embedded-atom method potential for $\mathrm{Ni}$ as described by Mishin et al. [20] was chosen in the present work because it is calibrated according to $\mathrm{ab}$ initio values of stacking fault and twin formation energies [20]. 
After the initial construction, energy minimization using the conjugate gradient method was performed to obtain the equilibrium configurations. The wires were then thermally equilibrated to $2 \mathrm{~K}$ for $20 \mathrm{ps}$ using a Nosé-Hoover thermostat $[21,22]$. No periodic boundary conditions were utilized in all three dimensions in this work.

Starting from the equilibrium configuration of the nanowires, uniaxial compressive loading was applied under the simulated quasistatic conditions $[7,11,14]$. To increase the simulation efficiency, in the first five loading steps all atoms were first displaced with a prescribed uniform compressive strain increment of $1 \%$ in the length direction and a $0.2 \%$ strain increment was applied in the remaining steps. The wires were then relaxed with their ends fixed at a constant temperature of $2 \mathrm{~K}$ for $50 \mathrm{ps}$ at their terminals to obtain a macroscopic equilibrium configuration. To ensure the simulated quasistatic condition, the equilibrium time for a loading step was determined by monitoring the stress of the whole system until there was little fluctuation. Typically, the equilibrium process usually took less than $150 \mathrm{ps}$ in the $16 \mathrm{~nm}$ wires. The average stress over the last $5 \mathrm{ps}$ of the relaxation period was taken as the stress of the nanowire. The stresses reported in this work were calculated using the Virial theorem, which is equivalent to the Cauchy stress in the average sense [23].

For the purpose of discerning defects in the nanowires, colors were assigned to the atoms according to a local crystallinity classification visualized by common neighbor analysis [24], which permits the distinction between atoms in a local hexagonal close-packed (hcp) environment and those in an fcc environment.

The obtained stress-strain curves for the three simulated wires are shown in Figure 1. The calculated Young's moduli are $\sim 300 \mathrm{GPa}$ and show no striking differences between the three wires. After the elastic deformation, the initial yielding results in precipitous stress drop under the displacement controlled loading. The yield stresses are 22.5, 26.8 and $29.3 \mathrm{GPa}$ for the 16, 8 and $4 \mathrm{~nm}$ wires, respectively. The following peak stresses after initial yielding of the three wires are 5.6, 10.2 and $15.1 \mathrm{GPa}$, respectively, also showing size effects (Fig. 1b), i.e. the magnitude of compressive yield stress decreases as the nanowire width increase. These size effect trends are in good agreement with the experimental reports in the literature [25], in which the diameterdependent yield stress of gold nanowires with size ranging from 40 to $200 \mathrm{~nm}$ measured by means of bending experiments was reported [25]. The yield stresses of $\mathrm{Ni}$ nanowires reported here are substantially greater than that of bulk Ni, but close to the theoretical stress, which is $30 \mathrm{GPa}$ from experiments [26].

After the incipient yielding, dislocations move out of the wires and cause a starvation state. Elastic deformation then continues until the second yielding. These processes repeat and result in a zigzag-like stress-strain response (see Fig. 1). Figure 2 shows the representative detailed stress variation as a function of relaxation time starting from the onset of the first yielding in the $8 \mathrm{~nm}$ nanowire. The calculated stress is recorded by averaging the stress within the relaxed period, i.e. 4 ps for every period, in the whole wires system. Close inspection of
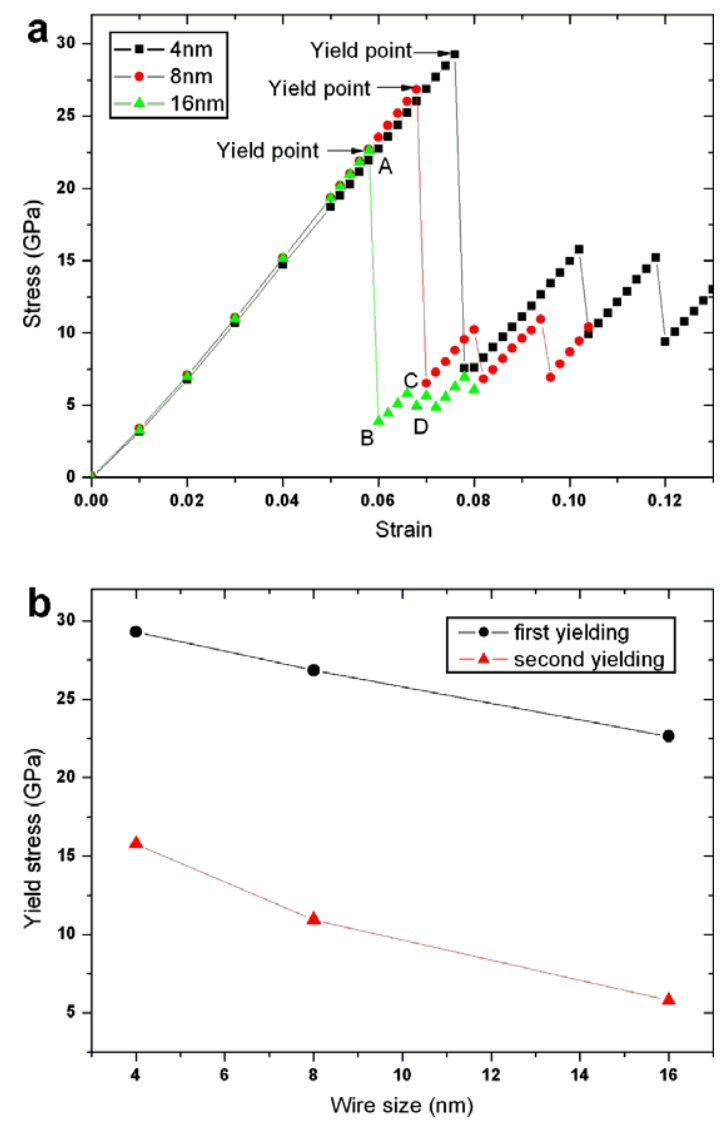

Figure 1. Compressive stress-strain curves of the nanowires ranging in size from 4 to $16 \mathrm{~nm}$.

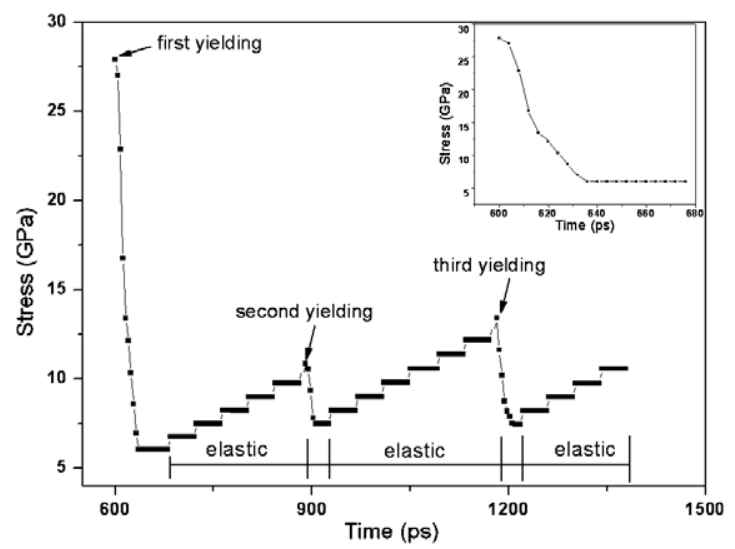

Figure 2. Representative stress vs. time curve of the $8 \mathrm{~nm}$ nanowire starting from the onset of first yielding, showing the plastic deformation separated by periods of elastic loading. The inset shows the enlarged stress vs. relaxation time curve during the first yielding.

stress as a function of relaxation time shows that the plastic deformation region, separated by periods of elastic deformation, corresponds to a significant drop in stress which continues with relaxation time, as shown in Figure 2.

A typical example of collective dislocation dynamics process in the $16 \mathrm{~nm}$ wires is shown in Figure 3. For the purpose of viewing, perfect fcc atoms are not shown in all these figures. These snapshots clearly demonstrate 


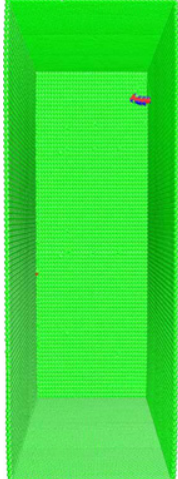

(a) $8 \mathrm{ps}$

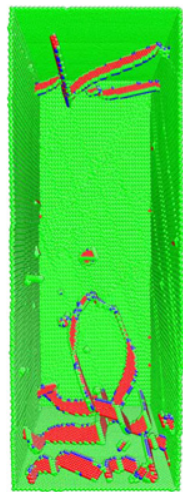

(d) $60 \mathrm{ps}$

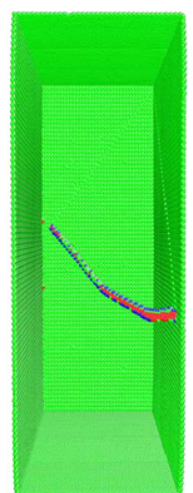

(b) $20 \mathrm{ps}$

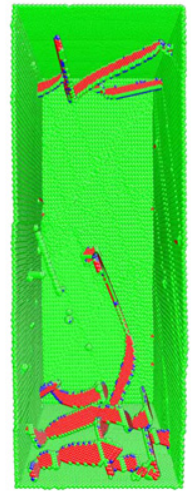

(e) $92 \mathrm{ps}$

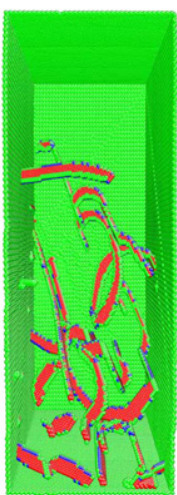

(c) $36 \mathrm{ps}$

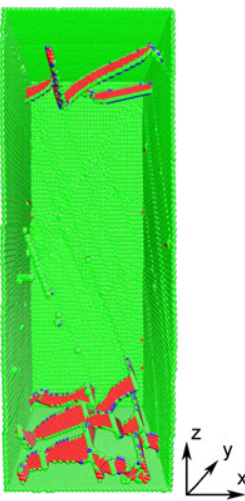

(f) $120 \mathrm{ps}$
Figure 3. Snapshots of first yielding deformation in $16 \mathrm{~nm}$ nanowire, showing collective dislocation movement in the wires corresponding to the relaxation process from point $A$ to point $B$ in Figure 1. The reference time occurs at the elastic loading prior to first yielding. Perfect fcc atoms and the front surface are not shown for viewing inner defects.

that dislocations are nucleated from surface edges. Once dislocations are nucleated from the surface edges, they can only travel much shorter distances before being annihilated at the free surface, thereby reducing the probability of multiplication processes.

The first compressive yielding of [111] nanowire is caused by the nucleation and propagation of $\frac{1}{2}\left[\begin{array}{lll}1 & 1 & 0\end{array}\right]$ full dislocation, regardless of the wire size, as shown in Figure 3. The Schmid factor analysis shows that slip of full dislocations would be crystallographically preferred over partial slip and twinning. Because the Schmid factor for a trailing partial dislocation is higher $(0.31)$ than the leading one $(0.16)$ in a [1 111$]$-oriented wire under compression, the trailing partial dislocation simultaneously follows the leading one and then creates the observed full dislocation.

Due to the finite scale in the length direction, some dislocation movement is blocked by the fixed end of the wires, which causes dislocation pile-up on the fixed boundaries, as shown in Figure 3f. After the dislocations leave the wires, multiple slip lines are clearly left on the free surfaces.

It should be noted that the first yielding always left dislocation slip lines on the surfaces, which are the most favorable sites for dislocation nucleation, and correspondingly the required stress for the second yielding is much lower than for the initial one (Fig. 1a). A typical dynamic dislocation process in the $16 \mathrm{~nm}$ wire during the second yielding is shown in Figure 4. This suggests that dislocation nucleation occurs preferentially near non-uniformities.

The mechanism of stress increase with strain after yielding in nanowires is much different to the strainhardening effects in conventional bulk metals, in which dislocations interact with each other and pile up at the grain boundaries, thereby requiring higher stresses with increasing strain. Evidently, plastic deformation in single-crystalline nanowires, where mobile dislocations can easily escape from the free surfaces before multiplying and interacting with other dislocations during the deformation process, differs in many respects from bulk metals. The simulations and analysis here highlight a significant difference between the behavior of discrete dislocations and the ensemble of dislocations that govern macroscopic metal plasticity. The compressive yielding of nanowires is caused by both the externally applied force and the surface-induced interior stresses [9-11]. The yielding criterion is still a topic of considerable interest to interpreting the size-dependence of the initial yielding. Diao et al. [11] stated that the averaged resolved shear stress (RSS) in the interior of a nanowire, which is caused by both the surface stress and the external applied force, can serve as the yielding criterion. The authors reported that the tensile yield stress of $\left[\begin{array}{ll}1 & 0\end{array}\right] \mathrm{Au}$ nanowires of 2-5 nm diameter increases with decreasing wire size but the compressive yield stress decreases with deceasing wire size. They attributed these opposite sizedependent yield stress trends to the effects of surface stresses, in which small wires exhibit large surface-induced internal compressive stresses [11]. For tensile yielding, a larger external force is required to make the nanowire yield in smaller wires. However, for compressive yielding, a smaller external force is needed in the small wires. We note that, due to non-linear elastic deformation behavior and different slip systems being activated in tensile and compressive loading of [100] wires $[10,11]$, the size-dependence of the yield stress of

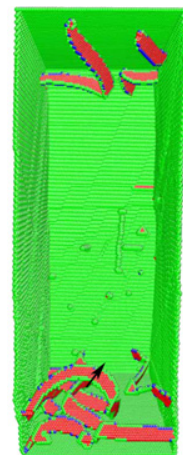

a

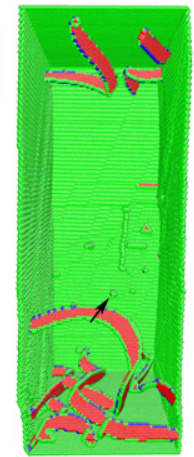

b

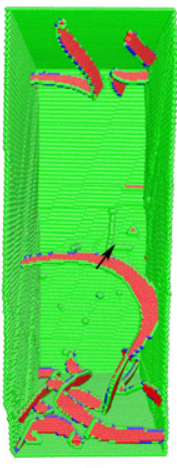

C

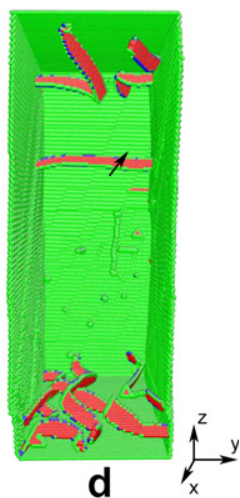

Figure 4. Snapshots of second yielding deformation in $16 \mathrm{~nm}$ nanowires, illustrating dislocation nucleation from previous left dislocations on the fixed boundary, propagation across the whole wire and then escape from the wire during second yielding from point $\mathrm{C}$ to point $\mathrm{D}$ in Figure 1. (a-d) Snapshots recorded with relaxation time. Perfect fcc atoms and the front surface are not shown for viewing inner defects. Black arrows indicate dislocation movement direction. 
[100] nanowires is not representative of other orientations. Furthermore, the local stress distribution near free surfaces is more important than the average stresses in the wire interior in controlling the yielding behavior of metallic nanowires.

For illustration, the detailed distribution of atomic level RSS [11] in an observed slip direction on an observed slip plane is shown in Figure 5. For the sake of clarity, atoms with RSS above the critical value $(5.0 \mathrm{GPa})$ are not shown. Only dislocation and free surfaces associated with low RSS are shown. The result indicates that the local critical RSS criterion does indeed predict the dislocation nucleation and propagation well at low temperature (here $T=2 \mathrm{~K}$ ). This also suggests that the criterion of dislocation nucleation is more closely associated with the local RSS distribution (corresponding to the local atomic structure) near the surface than the average value of the RSS in the interior of the wires. It is reasonable to consider that the size-dependent yield stress is an intrinsic property of nanowires and should not be dependent of loading condition (tensile or compressive).

In summary, we have performed atomistic simulations to study the mechanical behavior of defect-free singlecrystal $\mathrm{Ni}$ nanowires under compressive loading. We focused on elucidating the dislocation dynamics in nanowires with diameters ranging from 4 to $16 \mathrm{~nm}$. Alternating dislocations nucleate and propagate across the wire cross-section and then move out of the wires, and this leads to a state of dislocation starvation. Subsequently, elastic strain builds up stress until next yielding. Disloca-
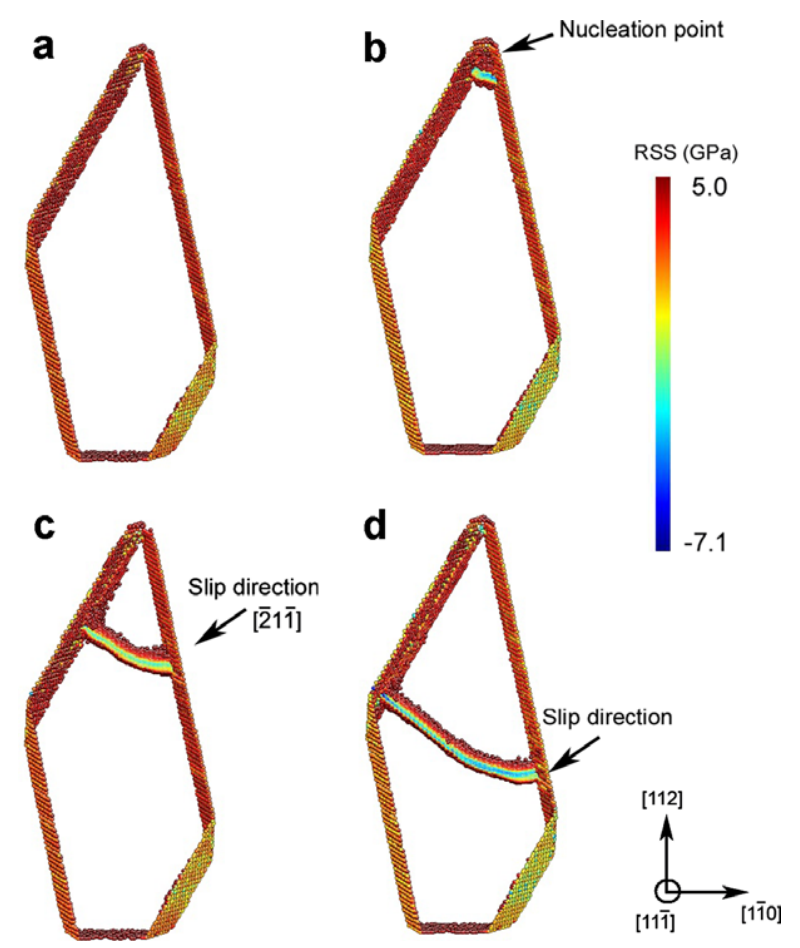

Figure 5. Distribution of the atomic level RSS in an observed slip direction on an observed slip plane in the course of initial yielding of the $16 \mathrm{~nm}$ wires. (a) Just prior to compressive yielding. (b) Dislocation nucleation from the free surface edge. (c, d) During the slip process. The atoms with RSS above the critical value $(5.0 \mathrm{GPa})$ are removed for viewing purposes. tion nucleation from previous defects, such as residual slip lines and dislocations piled-up on the fixed boundaries, would then be the dominant processes and correspondingly lower stress is required for dislocation nucleation in the later yielding. The aforementioned issues lead to the distinctly different mechanical behaviors in nanowires relative to bulk metals. In addition, the results show a size effect over the simulated size range: smaller is stronger, regardless of the loading condition (tensile/ compress). This work provides atomic-level evidence for the proposed dislocation starvation model, which can help gain fundamental insight into the size effects of plasticity in single-crystal nanowires (nanopillars).

The authors are supported by the National Science Foundation of China through Grant Nos. 10432050, 10721202 and 10672163 . We acknowledge S. Plimpton from Sandia National Laboratory for sharing his MD code ParaDyn [27].

Supplementary material associated with this article can be found, in the online version, at doi:10.1016/ j.scriptamat.2008.03.010.

[1] E.W. Wong, P.E. Sheehan, C.M. Lieber, Science 277 (1997) 1971.

[2] A. Hasmy, E. Medina, Phys. Rev. Lett. 88 (2002) 096103.

[3] E.Z. da Silva, A.J.R. da Silva, A. Fazzio, Phys. Rev. Lett. 87 (2001) 256102.

[4] Y. Kondo, K. Takayanagi, Phys. Rev. Lett. 79 (1997) 3455.

[5] Y. Kondo, K. Takayanagi, Science 289 (2000) 606.

[6] W. Liang, M. Zhou, F. Ke, Nano Lett. 5 (2005) 2039.

[7] W. Liang, M. Zhou, Phys. Rev. B 73 (2006) 115409.

[8] H.S. Park, K. Gall, J.A. Zimmerman, Phys. Rev. Lett. 95 (2005) 255504

[9] J. Diao, K. Gall, M.L. Dunn, Nat. Mater. 2 (2003) 656.

[10] J. Diao, K. Gall, M.L. Dunn, Nano Lett. 4 (2004) 1863.

[11] J. Diao, K. Gall, M.L. Dunn, J.A. Zimmerman, Acta Mater. 54 (2006) 643.

[12] K. Gall, J. Diao, M.L. Dunn, Nano Lett. 4 (2004) 2431.

[13] A. Cao, Y. Wei, Phys. Rev. B 74 (2006) 214108.

[14] A.J. Cao, Y.G. Wei, S.X. Mao, Appl. Phys. Lett. 90 (2007) 151909

[15] M.D. Uchic, D.M. Dimiduk, J.N. Florando, W.D. Nix, Science 305 (2004) 986.

[16] D.M. Dimiduk, C. Woodward, R. Lesar, M.D. Uchic, Science 312 (2006) 1188.

[17] J.R. Greer, W.C. Oliver, W.D. Nix, Acta Mater. 53 (2005) 1821.

[18] J.R. Greer, W.D. Nix, Phys. Rev. B 73 (2006) 245410.

[19] Z.W. Shan, R.K. Mishra, S.A. Syed Asif, O.L. Warren, A.M. Minor, Nat. Mater. 7 (2008) 115.

[20] Y. Mishin, D. Farka, M.J. Mehl, D.A. Papaconstantopoulos, Phys. Rev. B 59 (1999) 3393.

[21] S. Nosé, Mol. Phys. 52 (1984) 255.

[22] W.G. Hoover, Phys. Rev. A 31 (1985) 1695.

[23] J.D. Honeycutt, H.C. Andersen, J. Phys. Chem. 91 (1987) 4950.

[24] K.S. Cheung, S. Yip, J. Appl. Phys. 70 (1991) 5688.

[25] B. Wu, A. Heidelberg, J.J. Boland, Nat. Mater. 4 (2005) 525.

[26] A. Kelly, N.H. Macmillan, Strong Solids, second ed., Clarendon Press, Oxford, 1986.

[27] S.J. Plimpton, J. Comput. Phys. 117 (1995) 1. 